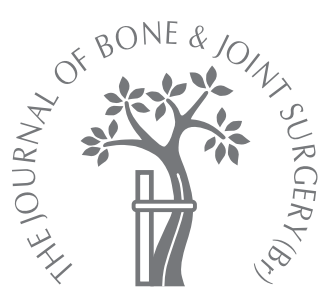

D. Donati,

A. El Ghoneimy,

F. Bertoni,

C. Di Bella,

M. Mercuri

From The Rizzoli

Orthopaedic

Institute, Bologna, Italy

\footnotetext{
D. Donati, MD, Consultant

C. Di Bella, MD

M. Mercuri, MD, Professor

Orthopaedic Division

F. Bertoni, MD, Professor

Department of Pathology

A. El Ghoneimy, MD,

Research Fellow

Department of

Musculoskeletal Oncology Istituto Ortopedico Rizzoli,

Via Pupilli 1, 40136 Bologna, Italy.

Correspondence should be sent to Dr D. Donati; e-mail: davide.donati@ior.it

(c)2005 British Editorial

Society of Bone and

Joint Surgery

doi:10.1302/0301-620X.87B11.

$16621 \$ 2.00$
}

$J$ Bone Joint Surg [Br]

2005;87-B:1527-30.

Received 15 April 2005

Accepted after revision

5 July 2005

\title{
Surgical treatment and outcome of conventional pelvic chondrosarcoma
}

\begin{abstract}
We reviewed 124 patients with a conventional pelvic chondrosarcoma who had been treated over a period of $\mathbf{2 0}$ years. We recorded the type of tumour (central or peripheral), type of operation (limb salvage surgery or hemipelvectomy), the grade of tumour, local recurrence and/or metastases, in order to identify the factors which might influence survival.

More satisfactory surgical margins were achieved for central tumours or in those patients treated by hemipelvectomy. However, grade 1 tumours, whatever the course, did not develope metastases or cause death, while grade 3 tumours had the worst outcome and prognosis.

Central, high-grade tumours require aggressive surgical treatment in order to achieve adequate surgical margins, particularly in those lesions located close to the sacroiliac joint. By contrast, grade 1 peripheral chondrosarcomas may be treated with contaminated margins in order to reduce operative morbidity, but without reducing survival.
\end{abstract}

Chrondrosarcoma is the second most common primary malignant tumour of bone after osteosarcoma. It occurs most often in the pelvis, the ilium being the most frequently involved bone, followed by the pubis and ischium. ${ }^{1-10}$

Because chemotherapy and radiotherapy do not influence the prognosis, the goal of surgery, which can be either a hindquarter amputation or an internal hemipelvectomy, is to resect the entire lesion with wide margins. ${ }^{10-12}$

Within the pelvis, there are no major anatomical barriers to extension of a tumour. Therefore, pelvic sarcomas can produce large, extraskeletal masses without specific symptoms other than pain. When patients finally seek medical advice, these tumours are often large. This, in addition to the proximity of important structures such as the bladder, rectum, and neurovascular bundles makes resection challenging. ${ }^{6,13,14}$ Local recurrence is frequent although the impact on the survival of the patient is unclear. However, it is reasonable to conclude that the prognosis is worse if there is recurrence.

We reviewed our experience of the surgical treatment of 124 intramedullary (central) and secondary (peripheral) pelvic chondrosarcomas. With adequate follow-up we investigated the factors which might influence the prognosis.

\section{Patients and Methods}

We reviewed the records of 124 patients with a pelvic chondrosarcoma who had presented between 1971 and 1999 and obtained data regarding the age, gender, date of diagnosis, previous treatment, the type and grade of the tumour, the date and type of surgery performed, surgical margins, complications and management, and the oncological outcome.

All available radiographs were reviewed for the following features: location of the tumour relative to the cortex (central or peripheral), the epicentre (the bone from which the tumour was thought to have arisen), the type of surgical intervention (limb-sparing resection or hemipelvectomy) and the site of local recurrence.

We included patients with a recurrence after previous surgery undertaken elsewhere. The volume of the tumour was not recorded. Each case was reviewed by a pathologist (FB), including the biopsy, the resection specimen, and any further specimens if there had been recurrence.

Conventional intramedullary and secondary chondrosarcomas were classified into three grades (1, 2 and 3). Dedifferentiated and mesenchymal chondrosarcomas were not considered since they are high-grade tumours. The only case of a clear-cell chondrosarcoma was also not included since it is a low-grade tumour. For 29 patients $(23.4 \%)$, their first operation had been in another institution and they had then been referred after a local recurrence. Ten of these $(34.5 \%)$ underwent hindquarter amputation at our hospital. 
Table I. The distribution of central (63) and peripheral (61) chondrosarcomas based upon the grade, site, and mean duration of pain by number and percentage

\begin{tabular}{|c|c|c|c|}
\hline & Central & Peripheral & p value ${ }^{*}$ \\
\hline \multicolumn{4}{|l|}{ Grade } \\
\hline 1 & $1(2)$ & $39(64)$ & \multirow{3}{*}{$<0.0005$} \\
\hline 2 & $44(70)$ & $22(36)$ & \\
\hline 3 & $18(28)$ & $0(0)$ & \\
\hline \multicolumn{4}{|l|}{ Site } \\
\hline Acetabulum & $31(49)$ & $4(7)$ & \multirow{5}{*}{$<0.0005$} \\
\hline Ilium & $11(18)$ & $33(54)$ & \\
\hline Pubis/ischium & $18(28)$ & $19(31)$ & \\
\hline Sacroiliac joint & $3(5)$ & $5(8)$ & \\
\hline Mean duration of pain (mths) & 19.5 & 8.2 & \\
\hline
\end{tabular}

* chi-squared, Pearson analysis

Table II. The grade and incidence of distant metastases, by number and percentage

\begin{tabular}{llll}
\hline & Grade 1 & Grade 2 & Grade 3 \\
\hline Pulmonary metastases & 0 & 1 & 7 \\
Other metastases & 0 & 2 & 0 \\
Total & $0(0)$ & $3(4.5)$ & $7 \quad(38.8)$ \\
\hline
\end{tabular}

Intra-operative margins were classified as 'wide', 'wide contaminated', 'marginal' and 'intralesional'. Only wide margins were considered to be adequate; contaminated margins were regarded as positive. The criteria for contaminated margins were related to the surgeon's operative description. If the tumour was violated during resection, even if additional tissue was removed to achieve wider margins, margins were considered to be wide contaminated. Consequently, the final decision about margins was only achieved after obtaining a consensus among surgeons, and after review of the pathology.

The date, site, and treatment of recurrent and metastatic lesions were recorded and the follow-up data obtained from the records were updated by a questionnaire sent to the patients.

Statistical analysis. This was performed using a chi-squared contingency table, Kaplan-Meier survival curves and multivariate Cox regression analysis. Values for $\mathrm{p} \leq 0.05$ were regarded as significant.

\section{Results}

A conventional chondrosarcoma was present in 124 patients; 63 tumours were central and 61 peripheral. The distribution according to the grade, site and the mean duration of pain for both types is shown in Table I. The epicentre for $31(49 \%)$ of the central tumours was located in the acetabulum, whereas for peripheral tumours, in 33 $(54 \%)$ it was in the ilium. This difference was statistically significant $(\mathrm{p}<0.0005)$. There was also a significant difference in severity of the tumours between the two groups with $44(70 \%)$ of central tumours being grade 2 and 39 $(64 \%)$ of peripheral tumours being grade $1(\mathrm{p}<0.0005)$.

We treated 44 central tumours by limb-sparing resection and $32(73 \%)$ had wide margins. Eighteen patients with central tumours underwent hemipelvectomy, and 14 (78\%) had wide margins. We treated 49 peripheral tumours by limb-sparing resection, and $25(51 \%)$ had wide margins. Twelve patients with peripheral tumours underwent hemipelvectomy, and ten $(83 \%)$ had wide margins. Central tumours had a wide margin more often than peripheral tumours, wide margins being obtained more frequently with hemipelvectomy than with limb-sparing resection $(\mathrm{p}=0.059, \mathrm{p}=0.077$, respectively). However, the type of operation did not correlate with the local rate of recurrence.

The tendency for tumours which crossed the sacroiliac joint to have inadequate margins, compared with the adequate margins of acetabular tumours was statistically significant $(\mathrm{p}=0.05)$. However, the differences in location of the tumour did not appear to influence the rate of local recurrence. Tumours located in the lower part of the pelvis (pubis and ischium) were more likely to be treated by hemipelvectomy $(\mathrm{p}=0.073)$.

For those patients with central tumours, the margins were wide in $46(73 \%)$ and positive in $17(27 \%)$. Of those with wide margins, eight $(17 \%)$ developed a local recurrence and six $(13 \%)$ died from disease. Of the 17 patients with inadequate margins, seven $(41 \%)$ had a local recurrence. For those with peripheral tumours, the margins were wide in $35(57 \%)$ and inadequate in $26(43 \%)$; of those with wide margins, only one $(3 \%)$ developed a local recurrence whereas six $(23 \%)$ patients with inadequate margins had a local recurrence. The lower incidence of recurrence in patients with wide rather than inadequate margins was statistically significant $(\mathrm{p}=0.016)$. There was local recurrence in five $(13 \%)$ of the patients with a grade $1,12(18 \%)$ with a grade 2 and five (28\%) with a grade 3 tumour. The relationships between the grade of tumour and local recurrence, and the grade of tumour and margins, were not statistically significant $(\mathrm{p}=0.187$ and $\mathrm{p}=0.367$, respectively). However, a significantly higher number of patients with grade 3 tumours underwent a hemipelvectomy $(\mathrm{p}<0.0005)$.

Table II shows the incidence of distant metastases for the different grades of chondrosarcoma. Higher grades were associated with a higher incidence of metastases $(\mathrm{p}<0.0005)$. No patient with a grade 1 chondrosarcoma developed distant metastases. For the higher grades, local recurrence was related to the incidence of metastases $(\mathrm{p}=0.016)$.

Survival analysis. The Kaplan-Meier curve for overall survival (Fig. 1) showed a mean survival of $92 \%$ at five years and $88 \%$ at ten years. However, patients with a peripheral chondrosarcoma have a better long-term survival $(96 \%$ at final follow-up) than those with a central chondrosarcoma $(73 \%)$. This difference was significant $(\mathrm{p}=0.00093)$. The overall survival related to the location of tumour is relative to the cortex shown in Figure 2, and the survival related to the grade in Figure 3, the grade being statistically related to survival. The difference in survival between grade 1 and grade 2 tumours was not significant $(p=0.06)$ although 


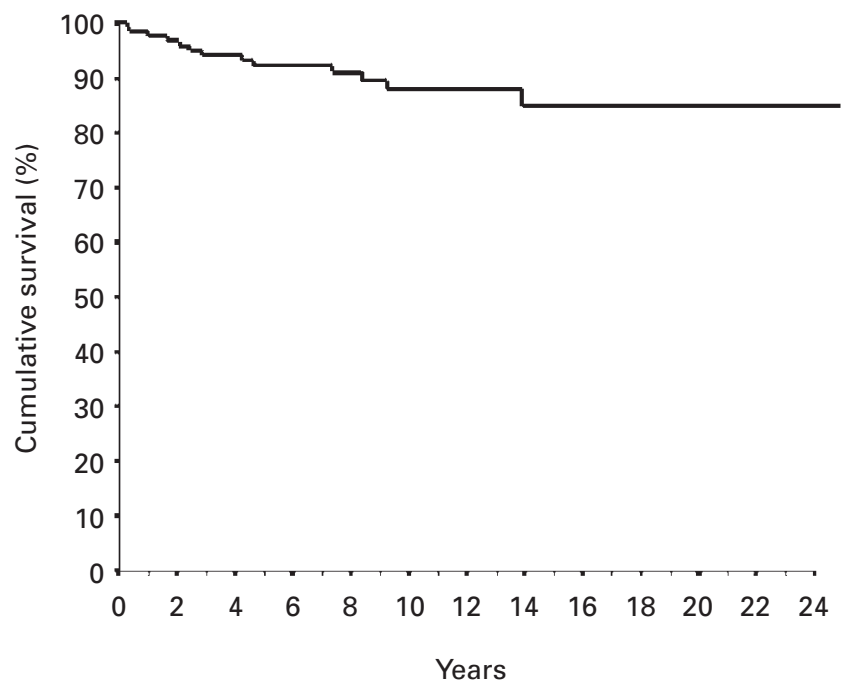

Fig. 1

Cumulative survival for the whole group of 124 patients.

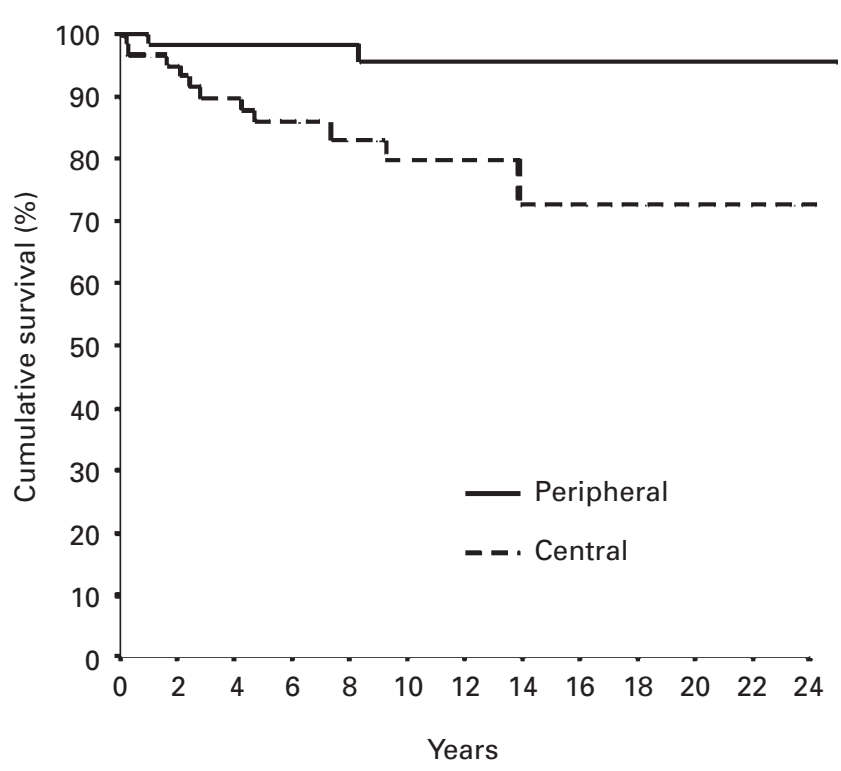

Fig. 2

Cumulative survival in the two groups of patients: peripheral and central pelvic chondrosarcoma.

both were associated with a higher overall survival than for grade 3 tumours $(\mathrm{p}<0.00005)$.

Only one patient was treated by curettage, while 30 underwent hemipelvectomy and 93 had a limb-sparing surgical resection. There was a better chance of survival in those treated by resection $(\mathrm{p}<0.00005)$.

Local recurrence had a significant effect on survival. Only $48 \%$ of those with a local recurrence were alive by the follow-up at 14 years while those without a local recurrence had a $96 \%$ chance of survival after ten years. Patients

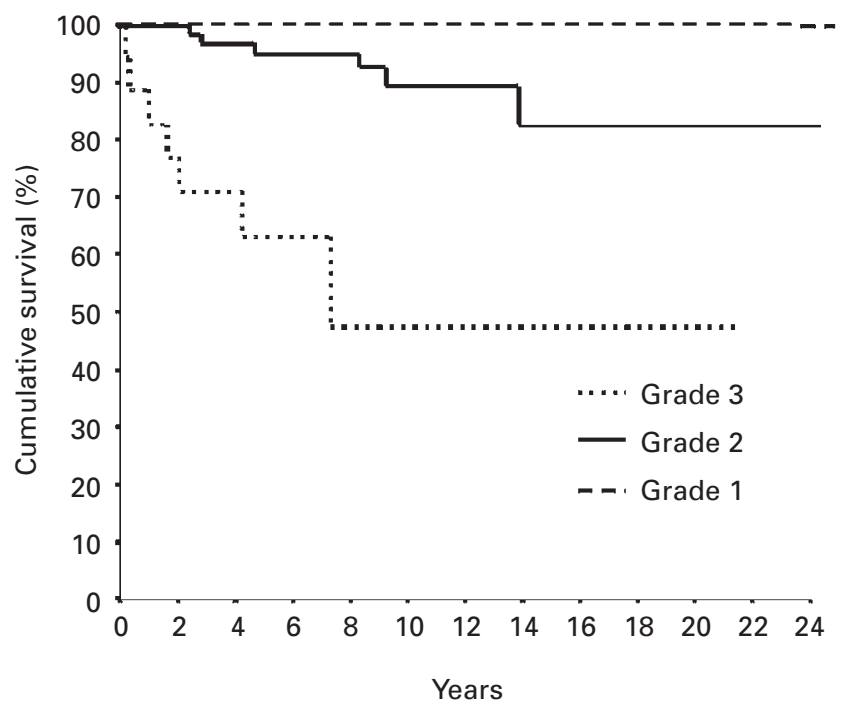

Fig. 3

Cumulative survival based upon the grade of tumour. No patient with grade 1 chondrosarcoma died from their disease.

with grade 1 and grade 2 tumours and with a local recurrence, had a five-year survival of $70 \%$ or more. However, those with a grade 3 chondrosarcoma who also had a local recurrence, had a five-year survival of less than $10 \% \mathrm{com}-$ pared with the five-year survival of $63 \%$ for all patients with grade 3 tumours. Multivariate analysis confirmed that the worst prognosis was related to the type of operation $(\mathrm{p}=0.0002 ;$ odds ratio, $30 ; 95 \%$ confidence interval $(\mathrm{CI}), 5$ to 189 ), local recurrence ( $\mathrm{p}<0.0000$; odds ratio, $43 ; 95 \%$ $\mathrm{CI}, 7$ to 267 ) and metastasis ( $<<0.0000$; odds ratio, 19 ; $95 \%$ CI, 4 to 78$)$.

Progression of grade. When reviewing the pathological specimens for patients with a local recurrence of grade 1 and grade 2 tumours, a higher grade (grade 3 ) was identified in three of 12 patients with a recurrent grade 2 chondrosarcoma. One of these patients presented with skeletal metastases shortly after their local recurrence and then died. No patient with a recurrent grade 1 tumour had a high-grade chondrosarcoma at the time of their recurrence.

\section{Discussion}

As noted by others, ${ }^{1}$ any system of grading for chondrosarcomas is intrinsically subjective, although grading is very important prognostically. When reviewing the pathology we considered both the surgeon's and pathologist's views in order to clarify best the impact of grade and surgical treatment on outcome.

In contrast to earlier studies, ${ }^{10,15}$ our analysis showed that the incidence of wide margins was much higher after hemipelvectomy than after a limb-sparing resection. This has been reported previously by Marcove et $\mathrm{al}^{16}$ in a study which predated current advances in imaging studies and limb salvage. The same could be said of our results, as many of our patients were treated in the early 1980s. How- 
ever, the influence of surgical margins, whether after hemipelvectomy or limb-sparing resection, had a significant effect on the rate of local recurrence. In our present study, the higher-grade tumours had more local failures although this was not statistically significant. Of the five local recurrences in grade 1 tumours, only one patient had wide margins at the time of resection, as opposed to four of the 12 patients with recurrent grade 2 tumours, and four of the five patients with recurrent grade 3 tumours. In an earlier report by Sheth et al, ${ }^{10}$ grade 1 pelvic tumours were locally less aggressive and there was a significant difference in the local recurrence-free survival between patients with grade 1 tumours and positive resection margins compared with grade 2, grade 3 and dedifferentiated chondrosarcomas with positive resection margins. Kreicbergs et $\mathrm{al}^{17} \mathrm{did}$ not find the histological grade to be a significant factor. It thus appears that the influence of the grade of chondrosarcoma on local failure is not, as yet, properly established.

Another important prognostic factor on local outcome is the site of the tumour. Those tumours which crossed the sacroiliac joint had a higher incidence of local recurrence compared with those at other sites. The inaccuracy of estimating the degree of tumour extension across the sacroiliac joint, as well as the difficulty in performing the bony cuts during resection combined with tumour infiltration into the sacral wing, explains the higher incidence of inadequate margins and local failure in these patients. Peripheral tumours showed a tendency towards inadequate margins compared with central lesions. This may be attributed to the larger size of peripheral tumours although we were unable to include the volume of the tumour in our study since the relevant information was not available for most of our patients.

The single, most significant prognostic factor for distant failure was the grade of the tumour. No patient with a grade 1 tumour developed distant metastases, and none died from their disease. Local recurrence was not associated with an increased incidence of metastases in either grade 1 or grade 2 tumours, with only one patient in the latter group developing distant metastases.

The relationship between local failure and overall survival in chondrosarcoma has been extensively reported. Sheth et $\mathrm{al}^{10}$ stated that local recurrence did not influence overall survival in their series. However, Ozaki et $\mathrm{a}^{11}$ studied the outcome of 26 patients treated by intralesional surgery. The overall survival for patients with grades 1 and 2 (combined) or grade 3 chondrosarcomas was $85 \%$ and $44 \%$, respectively. According to Lee et al, ${ }^{9}$ local recurrence had no effect on the rate of either metastases or death in their group of patients with low-grade chondrosarcomas.

In our study, the survival of patients with a pelvic chondrosarcoma was greatly influenced by the grade of tumour because of the higher incidence of distant metastases with higher tumour grades. Local recurrence did not significantly affect the survival for low-grade compared with high-grade tumours since this was not associated with an increased incidence of distant metastases although there was an increased morbidity. Adequate surgical margins are essential in the treatment of pelvic chondrosarcoma in order to reduce the incidence of local failure and distant metastases. Weber et $\mathrm{al}^{14}$ recently reported the outcome of recurrent chondrosarcoma of the pelvis. In $14 \%$ (three cases), the tumour at the initial recurrence was of a higher grade than at first presentation. In six additional cases it became a higher grade during subsequent recurrences. In our study, no patient with a grade 1 tumour had a higher grade at the time of their recurrence, although $25 \%$ of patients with grade 2 tumours had a higher grade at the time of recurrence.

In conclusion, the treatment for a conventional pelvic chondrosarcoma can be either by limb-sparing resection or hemipelvectomy. Central, high-grade tumours require aggressive surgical treatment in order to achieve adequate surgical margins, particularly those located close to the sacroiliac joint. By contrast, grade 1 peripheral chondrosarcomas may be treated with contaminated margins in order to reduce the incidence of operative and post-operative morbidity but without reducing the survival.

No benefits in any form have been received or will be received from a commercial party related directly or indirectly to the subject of this article.

\section{References}

1. Bjorrnsson J, Mcleod R, Unni K, Ullstrup D, Pritchard DJ. Primary chondrosarcoma of long bones and limb girdles. Cancer 1998;83:2105-19.

2. Miller RW, Boice JD Jr, Curtis RE. Bone cancer. In: Schottenfeld D, Fraumeni JF Jr, eds. Cancer epidemiology and prevention. Second edition. New York: Oxford University Press, 1996:971-83.

3. Larsson SE, Lorentzon R. The geographic variation of the incidence of malignant primary bone tumours in Sweden. J Bone Joint Surg [Am] 1974;56-A:592-600.

4. Unni KK. Dahlin's bone tumors: general aspects and data on 11,087 cases. Fifth ed. Philadelphia: Lippincott-Raven, 1996:71-108.

5. Schajowicz F. Tumor and tumor like lesions of bone: pathology, radiology, and treatment. Second edition. Berlin: Springer-Verlag, 1994:141-244.

6. Huvos AG. Bone tumors: diagnosis, treatment, and prognosis. Philadelphia: W.B. Saunders Co., 1991:343-93.

7. Ozaki T, Hillmann A, Lindner N, Blasius S, Winkelmann W. Chondrosarcoma of the pelvis. Clin Orthop 1997;337:226-39.

8. Evans HL, Ayala AG, Romsdahl MM. Prognostic factors in chondrosarcoma of bone: a clinicopathologic analysis with emphasis on histologic grading. Cancer 1977; 40:818-31.

9. Lee FY, Mankin HJ, Fondren G, et al. Chondrosarcoma of bone: an assessment of outcome. J Bone Joint Surg [Am] 1999;81-A:326-38.

10. Sheth DS, Yasko AW, Johnson ME, et al. Chondrosarcoma of the pelvis: prognostic factors for 67 patients treated with definitive surgery. Cancer 1996;78:745-50.

11. Ozaki T, Lindner $\mathbf{N}$, Hillmann $\mathbf{A}$, et al. Influence of intralesional surgery on treatment outcome of chondrosarcoma. Cancer 1996;77:1292-7.

12. Healey JH, Lane JM. Chondrosarcoma. Clin Orthop 1986;204:119-29.

13. Monticelli G, Santori FS, Ghera S, Folliero A, Manili M. Surgical problems in the treatment of pelvic tumors. In: van Oosterom A, Van Unnik M, eds. Management of soft tissue and bone sarcomas. New York: Raven Press, 1986:101-6.

14. Weber KL, Pring ML, Sim FH. Treatment and outcome of recurrent pelvic chondrosarcoma. Clin Orthop 2002;397:19-28.

15. Kyoo-Ho S, Rougraff BT, Simon MA. Oncologic outcome of primary bone sarcoma of the pelvis. Clin Orthop 1994;304:207-17

16. Marcove RC, Miké V, Hutter RVP, et al. Chondrosarcoma of the pelvis and upper end of the femur: an analysis of factors influencing survival time in one hundred and thirteen cases. J Bone Joint Surg [Am] 1972;54-A:561-72.

17. Kreicbergs A, Boquist L, Borssen B, Larsson S. Prognostic factors in chondrosarcoma: a comparative study of cellular DNA content and clinicopathologic features. Cancer 1982:50:577-83. 